Apidologie, 1981, 12 (2), 159-183.

\title{
RASSENSPEZIFISCHE UNTERSCHIEDE IN OVARENTWICKLUNG UND EIABLAGE VON WEISELLOSEN ARBEITERINNEN DER HONIGBIENE APIS MELLIFERA L.
}

\author{
Friedrich RUTTNER und Bernd HESSE
}

Institut für Bienenkunde, Im Rothkopf 5, 6370 Oberursel, Bundesrepublik Deutschland

\section{ZUSAMMENFASSUNG}

Bei sieben Bienenrassen wurden bei weisellosen Arbeitsbienen Verlauf der Ovarentwicklung, Ovariolenzahl, Zeitpunkt des Beginns der Eiablage (Latenzzeit) und Zahl der in den ersten 48 Stunden abgelegten Eier unter gleichartigen Umweltbedingungen im freifliegenden Volk bestimmt.

A. $m$. carnica hatte mit 30 Tagen die längste, $A$. m. intermissa mit 5,6 Tagen die kürzeste Latenzzeit. Es ergaben sich drei deutlich unterscheidbare Gruppen - die europäischen Rassen Carnica, Ligustica und Mellifera mit einer Latenzzeit von 22-30 Tagen, Adami aus Kreta (ein anatolisches Element) mit 16 Tagen und die afrikanischen Rassen Intermissa, Scutellata (= "adansonii") und Capensis mit 5-10 Tagen.

Die Latenzzeit korreliert negativ mit der Zahl der abgelegten Eier, aber nicht mit der Zahl der Ovariolen.

Die Ergebnisse zeigen, dass die Fortpflanzungsfähigkeit der Arbeitsbienen nicht nur durch Umwelteinflüsse (Königin, offene Brut, Pollenernährung), sondern auch durch genetische Faktoren gesteuert wird.

\section{EINLEITUNG}

Bei sämtlichen sozial lebenden Hymenopteren beruht die soziale Struktur auf einem mehr oder weniger labilen Spannungszustand zwischen dem einzigen (oder den wenigen) voll reproduktionsfähigen Weibchen $(q)$ und den im Habitualzustand infertilen Arbeiterinnen ( $\zeta \zeta)$. Während der Entwicklung behalten nur bei der $\$$ die ursprünglich bei beiden Kasten gleich angelegten Gonaden ihren hohen Entwicklungsstand, während bei den $\not{\zeta} \not$ eine weitgehende Reduktion eintritt.

Bei einem "typischen" Bienenvolk - aber auch bei anderen sozialen Hymenopteren - ist festzustellen, dass die $q$ allein sämtliche Nachkommen liefert und 
dass die $\Varangle \ngtr$ in keiner Weise an der Reproduktion beteiligt sind. Dieser Zustand kann potentiell unbegrenzt anhalten. Eine Reproduktion von $\zeta \nvdash$ ist in diesem System nicht vorgesehen und anscheinend ohne erkennbare Bedeutung.

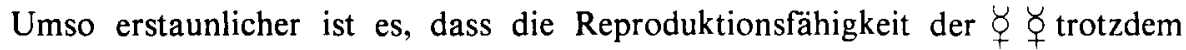
nicht völlig abgebaut wird. Es ist bisher bei sozialen Hymenopteren in keinem einzigen Fall der Nachweis erbracht worden, dass $\not{\zeta} \not$ unter bestimmten Bedingungen nicht in der Lage wären, entwicklungsfähige Eier abzulegen. Nicht selten geschieht dies sogar in der Anwesenheit einer fertilen Königin, vor allem wenn diese nicht in voller Funktion ist - so bei der Honigbiene (GonTARSKI, 1938), bei vielen Meliponinen (SAKagami et al., 1963) und bei Ameisen. Bei der Myrmicine Temnothorax treten legende $\Varangle \not$ nur auf, wenn sich die $q$ nicht in Eiablage befindet (z. B. nach der Überwinterung), später verschwinden sie wieder (DejEan u. PAsSERA, 1974).

Die Beibehaltung der potentiellen Reproduktionsfähigkeit der Bienen- $\not \zeta$ steht im Gegensatz zu der tiefgreifenden Differenzierung zwischen den beiden Kasten, die sich auf eine sehr grosse Anzahl von Merkmalen erstreckt (s. WEISs, 1980).

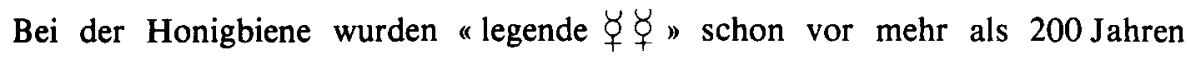
(RIEM, 1765) und seither in einer grossen Zahl von Arbeiten beschrieben. Der

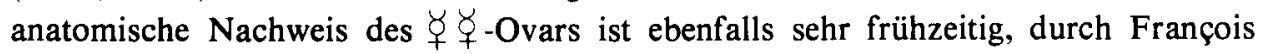
Huber's Mitarbeiterin JURINE (1814) und später durch LeUCKART, 1857 (s.v. BerLePSCH, 1870) erfolgt; Reaumur und Swammerdam erwähnen in ihren sehr sorgfältigen Untersuchungen dieses Organ hingegen nicht.

\section{Die Ovarentwicklung im Lebenszyklus der Arbeitsbiene}

In den frühen Stadien ist die Ovaranlage bei allen weiblichen Larven der Honigbiene gleich. In jeder der beiden Anlagen sind 180-200 Ovariolen vorhanden (ZANDER, LösChel u. MeIER, 1916). Im Zuge der Kastendetermination, ab dem 3. Larventag, tritt eine Differenzierung ein :

Bei der zur $q$ bestimmten Larve bleibt die ursprünglich angelegte Ovariolenzahl erhalten, ausserdem vergrössert sich die Spermatheka und es entwickeln sich die übrigen für die $q$ charakteristischen Merkmale (Zusammenfassung bei WeIss, 1980). Bei der zur $\Varangle$ determinierten Larve hingegen erfolgt eine während der ganzen Larvalentwicklung fortschreitende Reduktion der Ovariolenzahl bis auf den Imaginalwert.

Durch Ausseneinflüsse (z.B. Änderung der Ernährung während der Larvalentwicklung) kann die Zahl der Ovariolen nicht verändert werden (WEAVER, 1956). Dagegen führt eine Störung der $q$-determinierenden Faktoren (z. B. bei Aufzucht im Labor oder bei später Umstimmung) zu Zwischenformen zwischen $q$ und $\zeta$, wobei die Fortpflanzungsorgane (Ovarien, Spermatheka) früher betroffen werden als andere Merkmale. 
Nach Williams und Free, 1975 führt die Larvenaufzucht im weisellosen Volk zu $\Varangle \nvdash$ mit signifikant verstärkter Tendenz zur Ovarentwicklung.

Im allegemeinen sind die Ovarien frischgeschlüpfter $\not \zeta$ unentwickelt. Sie können sich aber bei jeder $\Varangle$ bis zur Ablage lebensfähiger Eier entwickeln, soferne nur die Grundvoraussetzungen dazu gegeben sind:

- Leben im Sozialverband;

- Adäquate Ernährung;

- Fehlen von Hemmfaktoren.

Eine geeignete Ernährung ist für beide Bienenkasten Voraussetzung für die Ablage von Eiern. Ohne Pollen- (bzw. Protein-) Versorgung kommt es bei den $\not \Varangle \not$ nicht zur Entwicklung der Ovarien (MüssBichleR, 1952).

Diese Beobachtung wird auch in der Praxis der Bienenhaltung ausgewertet : Ein Bienenvolk mit legenden $\Varangle \not$ lässt sich nur schwer durch Zusetzen einer $q$ in seinen Normalzustand zurückführen. Nach ÖRösı PAL (Zit. nach GoNTARSKI, 1938) verschwinden jedoch die legenden Arbeitsbienen sehr rasch, wenn man das Volk von seinen Waben in eine leere Kiste abkehrt und zwei Tage lang hungern lässt.

Physiologische Voraussetzung für die Entwicklung der Ovarien ist die Speicherung von Proteinen im Bienenkörper. Dreischer (1956) stellte fest, dass sich zuerst die Futtersaftdrüsen vergrössern, bevor die Ovarentwicklung einsetzt. Auf die Rolle des Juvenilhormons weist die Vergrösserung der Corpora allata bei $\not \ngtr \not$ mit entwickelten Ovarien hin.

Die Hemmung, der Ovarentwicklung bei den $\not \Varangle$ geht vor allem von der $q$ aus. Der Grad der Ovarentwicklung bei $\not{q} \not$ wird seit BuTLER 1954 allgemein als Biotest für den Faktor "Königin » benutzt. In einem Volk mit legetüchtiger, vitaler $q$ findet man höchstens bei einer geringen Anzahl von Bienen schwach entwickelte Ovarien obwohl durchaus auch im weiselrichtigen Volk legende $\not \gamma \not$ auftreten können (GONTARSKI, 1938; Müssbichler, 1952). Schon eine geringe Abschwächung des Einflusses der $q$, etwa durch Absperren durch ein für Bienen passierbares Gitter, führt zu einer messbaren Ovarentwicklung bei den $\not \not \zeta$. Andererseits haben selbst tote $\$ q$ längere Zeit hindurch einen hemmenden Einfluss (PAIN, 1954), ebenso Extrakte aus ganzen $q \oint$ (VoOGD, 1955) oder aus Mandibulardrüsen (BUTLER, 1959; GARY, 1961). Velthuis, 1970 benutzte die Hemmwirkung auf die $\not \Varangle \not$-Ovarien zum Nachweis der Funktion der Dermaldrüsen in den abdominalen Tergittaschen der $q$.

Auch legende $\Varangle \not$ hemmen bei den anderen $\Varangle \Varangle$ desselben Volkes die Entwicklung der Ovarien (SAKAGAMI, 1958; Velthuis, 1970). Bei der legenden KapArbeitsbiene, deren Mandibeldrüsen erhebliche Mengen der Hauptkomponente der "Queen Substance ", 9-oxo2-trans-Decensäure, produziert (RuTTNER, KoENIGER und Veith 1976; Hemmling, Koeniger und Ruttner 1979), ist die Hemmwirkung fast genauso stark wie bei einer $q$. Das kann in ganz einfachen Käfigversuchen überzeugend demonstriert werden (Tab. 1). 
TAB. 1. - Ovarentwicklung bei Arbeitsbienen ohne Königin, mit Königin oder mit einer legenden Kap-Arbeiterin.

TABL. 1. - Ovary development of worker bees without queen, with queen or with a laying Cape worker.

\begin{tabular}{|c|c|c|c|c|c|}
\hline $\begin{array}{c}\text { Ovarentwicklung } \\
\text { Development of ovaries }\end{array}$ & & $\begin{array}{l}\text { Stufe I } \\
\text { Class }\end{array}$ & $\begin{array}{l}\text { Stufe II } \\
\text { Class }\end{array}$ & $\begin{array}{l}\text { Stufe III } \\
\text { Class }\end{array}$ & $\mathbf{n}$ \\
\hline $\begin{array}{l}\text { Weisellos } \\
\text { Queenless }\end{array}$ & $\begin{array}{l}1976 \\
1977\end{array}$ & $\begin{array}{l}57 \\
71\end{array}$ & $\begin{array}{r}100 \\
51\end{array}$ & $\begin{array}{l}43 \\
30\end{array}$ & \\
\hline $\begin{array}{l}\text { Mit } q \\
\text { With } q\end{array}$ & $\begin{array}{l}1976 \\
1977 \\
\end{array}$ & $\begin{array}{l}128(36,4 \%) \\
165 \\
90\end{array}$ & $\begin{array}{c}151(42,9 \%) \\
6 \\
0 \\
\end{array}$ & $\begin{array}{l}73(20,7 \%) \\
0 \\
0\end{array}$ & 352 \\
\hline $\begin{array}{l}\text { Mit legender } \gamma^{\gamma} \\
\text { With laying }{ }^{\prime}\end{array}$ & $\begin{array}{l}1976 \\
1977\end{array}$ & $\begin{array}{l}255(97,6 \%) \\
172 \\
91\end{array}$ & $\begin{array}{l}6(2,4 \%) \\
6 \\
0\end{array}$ & $\begin{array}{l}0 \\
1 \\
0\end{array}$ & 261 \\
\hline & & $263(97,4 \%)$ & $6 \overline{(2,2 \%)}$ & $1(0,4 \%)$ & 270 \\
\hline
\end{tabular}

Ein weiterer sehr wirksamer Hemmfaktor für die Ovarentwicklung der $\not{q} \not$ ist das Vorhandensein von Larven (PerepelowA, 1926). MüssBichler (1962) konnte durch Zugabe von offener Brut zu weisellosen Völkern die Ovarien der $\not \not \not ̧$ auf einem sehr niedrigen Entwicklungsstand halten. Vielleicht ist die drastische Verringerung der Legetätigkeit der $q$ und damit der Larvenzahl mit ein Grund dafür, dass in Völkern, die sich im Schwarmstadium befinden, mehrfach eine verstärkte Ovarentwicklung bei $\not \Varangle$ gefunden wurde (Perepelova, 1929; Verheijen-Voogd, 1969; Velthuis, 1970). Allerdings besteht am Höhepunkt der Volksentwicklung, von Juni bis Mitte August, eine erhöhte Tendenz zur Ovarentwicklung, die im Herbst wieder stark abfällt (Hess, 1942; MüssBichler, 1952; Velthuis, 1970). Dieser Umstand muss bei der Durchführung vergleichender Versuche berücksichtigt werden (s. unten).

Nicht alle Eier, die sich in den Ovarien bis zur - morphologischen - Reife entwickeln, werden abgelegt. Die Eier können wieder resorbiert werden (Oosorption; Hess, 1942; GONTARSKı, 1938). Unvollständig resorbierte Eier können abgelegt werden, sie haben eine eigenartig geschrumpfte Gestalt und sind nicht entwicklungsfähig (Abb. 1).

Deshalb ist keine Aussage darüber möglich, ob eine $\Varangle$ mit Ovarien der Entwicklungsstufe 3 schon Eier abgelegt hat oder ob sie überhaupt zur Eiablage gelangen wird. DreisCher (1956) unterscheidet zwischen "morphologisch legenden $\Varangle \not$ " und " funktionell legenden $\not \zeta$ ".

In der vorliegenden Untersuchung werden beide Kriterien verwendet: Die tatsächliche Ablage von Eiern als der letzten Entwicklungsstufe und als eindeutiger Nachweis des Eintritts der $\Varangle \Varangle$ in die Reproduktionsphase. Und der anatomische Entwicklungszustand der Ovarien als Nachweis der Entwicklung in einem bestimmten Zeitabschnitt. 


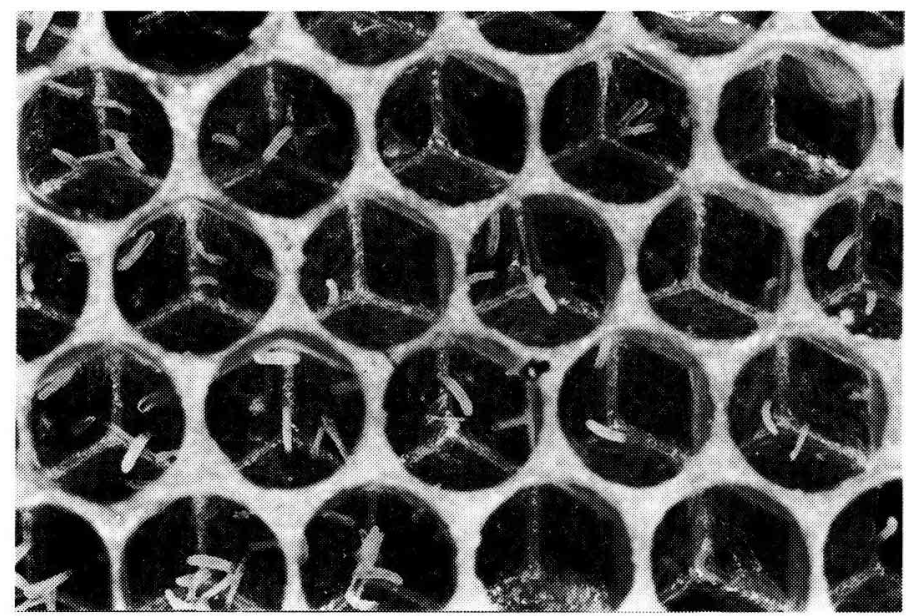

Aвв. 1. - Arbeiterinnen-Gelege (A. m. adami).

FIG. 1. - Worker laid eggs (A. m. adami).

Schon in den frühen Beobachtungen (z. B. bei BERLEPSCH, 1873) wird angegeben, dass vom Verlust der bis zur Ablage der ersten Eier durch $\not{\zeta} \not$ etwa 4-5 Wochen vergehen. Diese Beobachtungen beziehen sich auf europäische Rassen. Später wurde festgestellt, dass dieser Zeitabstand besonders bei afrikanischen Rassen auch wesentlich kürzer sein kann (Zusammenstellung bei RUTTNER, 1975). Es gab also Hinweise dafür, dass die Tendenz zur Entwicklung von funktionstüchtigen Ovarien nicht nur von Umweltfaktoren wie Ernährung oder der Abwesenheit einer $\subsetneq$ und Fehlen von Brut abhängt, sondern auch von angeborenen, genetisch bedingten Faktoren. Dasselbe gilt für die Anzahl von Ovariolen, die nach WeAver (1956) sogar bei verschiedenen Linien derselben Rasse verschieden sein kann.

Deshalb sollte die Frage nach genetisch bedingten Unterschieden in der Bereitschaft der $\not \zeta$ verschiedener Rassen zur Fortpflanzung und in der Zahl der Ovariolen in möglichst exakt kontrollierten Versuchen geprüft werden.

Wegen der Vielzahl geographischer Rassen, die unter völlig verschiedenen ökologischen Bedingungen leben, erscheint die Honigbiene als ideales Untersuchungsobjekt für diese Fragen.

\section{MATERIAL UND METHODEN}

Bei der Auswahl der Bienenrassen für diese Untersuchung spielte die Verfügbarkeit von lebendem Material eine dominierende Rolle. Es wurden aber grosse Anstrengungen unternommen, um aus für diese Frage besonders interessierenden Gebieten, das ist vor allem der afrikanische Kontinent, $q$ ? reiner Rassen $\mathrm{zu}$ beschaffen. Die Versuche wurden am Gelände des Instituts für Bienenkunde Oberursel durchgeführt. 
Folgende Rassen standen zur Vefügung :

1. Apis mellifera carnica PollmanN aus den österreichischen Alpen.

2. Apis mellifera ligustica SPINOLA aus dem Gebiet von Bologna.

3. Apis mellifera mellifera L. aus Südfrankreich (Provence).

4. Apis mellifera adami RuTTNER von der Insel Kreta.

5. Apis mellifera adansonii-scutellata LATreILlE aus Pretoria, SA.

6. Apis mellifera intermissa v. BUTTEL-REEPEN aus Tunesien.

7. Apis mellifera capensis Escholtz, aus der Kapprovinz, Südafrika.

Nach der zoogeographischen Zugehörigkeit handelt es sich um drei europäische Rassen (Nr. 1-3), um eine anatolische (Nr. 4) und um drei afrikanische Rassen (Nr. 5-7).

Arbeitsbienen dieser Rassen liessen wir in einem Brutschrank schlüpfen und setzten sie, um ihnen von vorneherein die bestmöglichen Bedingungen zu bieten, kleinen Carnica-Völkchen zu. Später entfernten wir die Carnica-Bienen und entweiselten die Völkchen, um nun die mit der Eierlegetätigkeit der Arbeitsbienen zusammenhängenden Fragen zu untersuchen.

Die Versuchsvölkchen wurden zunächst aus Kunstschwärmen mit etwa 1500 Carnica-Bienen gebildet, in Kästchen mit 3 Rähmchen $(10 \times 15 \mathrm{~cm})$ untergebracht und mit einer schlüpfreifen CarnicaWeiselzelle versehen. Nach drei Tagen Kellerhaft wurden die Kästchen auf einem durch Sträuchergruppen gut markierten Gelände in Gruppen zu je drei oder vier aufgestellt. Die Gruppen standen auf dem Versuchsgelände (Ausmass $80 \times 140 \mathrm{~m}$ ) in Abständen von mindestens $15 \mathrm{~m}$ voneinander entfernt. Die für die Capensis-Bienen vorgesehenen Völkchen wurden wegen der von dieser Rasse bekannten Verflugsneigung völlig isoliert in einer Entfernung von $500 \mathrm{~m}$ aufgestellt. Infolge der zeitlichen Versetzung der Versuche befanden sich nie mehr als vier Gruppen gleichzeitig auf dem Gelände. Tatsächlich konnte kein Verflug zwischen den Gruppen beobachtet werden.

Sieben Tage nach der Aufstellung hatten die Carnica- $q q$ die Hochzeitsflüge absolviert und sie begannen mit der Eiablage.

Die Versuchsbienen wurden durch Einstellen verdeckelter Brutwaben aus Völkern der ausgewählten Rasse in einem Brutschrank gewonnen. Täglich wurden die geschlüpften Bienen abgesammelt, mit einer tages - und rassenspezifischen Farbe markiert und den für diese Rasse vorgesehenen Völkchen zugesetzt.

Die tägliche Markierung einer so grossen Zahl von Jungbienen erforderte eine rationelle Technik : Die frischgeschlüpften Bienen wurden auf eine Styroporplatte geschüttet und mit einem durch ein Nylonnetz bespannten Rahmen bedeckt. Die durch das Gitter festgehaltenen Bienen liessen sich jetzt rasch und bequem mit einem Farbtupfer markieren. Die an einem bestimmten Tag geschlüpften Bienen einer Rasse wurden alle mit derselben Farbe markiert und auf 3 oder 4 Völkchen einer Gruppe aufgeteilt. Bienen verschiedener Rasse konnten somit unterschieden werden, innerhalb einer Rasse (Gruppe) war aber nur eine Unterscheidung nach dem Alter, nicht aber nach dem Kästchen möglich.

Jedem Volk wurden 14 Tage lang täglich 80-200 markierte Jungbienen zugesetzt. Nach dieser Zeit waren im Völkchen ebensoviele markierte Bienen wie Pflegebienen (etwa 1 500) vorhanden.

Die Völkchen wurden ständig mit Zuckerteig gefüttert. Die Versorgung mit Naturpollen während der Versuchszeit war gut. Bei Schlechtwetter wurde vorsorglich eine Honig-Pollenmischung verabreicht.

Drei Tage nach Beginn der Eiablage wurde die $q$ in ihrem Volk in einem Käfig gesperrt. Sechs Tage später, nach dem Zusetzen der letzten (14.) Gruppe markierter Jungbienen wurde der Käfig mit der $q$ entfernt, ebenso wurden alle nichtmarkierten Bienen im Volk mit einem Exhaustor abgesammelt und entnommen.

Die Versuchsvölkchen enthielten jetzt also etwa 1400 -1 500 markierte Bienen einer bestimmten Kasse im Alter von 1-14 Tagen ohne $q$, sowie ältere Larven von der entnommenen $q$. Nach der Entweiselung wurden anfangs Weiselbecher in verschiedener Zahl gebaut, aber für eine Weiselpflege waren die vorhandenen Larven schon zu alt. Drei Tage nach Entnahme der $q$ war auch die letzte Brutzelle verdeckelt; diese Brut war von grosser Bedeutung für den Zusammenhalt des Völkchens nach der Entweiselung. Elf Tage später begann die Brut zu schlüpfen; die hinzukommenden Jungbienen bewirkten eine Verstärkung der Völkchen, sie konnten wegen des grossen Altersabstandes aber den Versuchsablauf nicht stören, zumal sie der Carnica-Rasse mit langsamer Ovarentwicklung angehörten. 
Der zeitliche Ablauf des Versuchsaufbaues ist in Abb. 2 dargestellt.

Eine abweichende Behandlung erfuhren nur die Völkchen mit $A$. m. intermissa. Von dieser Rasse konnten jedem Völkchen nur 500-700 Bienen zugesetzt werden, da nicht ausreichend Brut vorhanden war. Deshalb wurden die nichtmarkierten Bienen belassen, um die Völkchen lebensfähig zu erhalten. Da gerade diese Rasse eine besonders kurze Latenzzeit aufweist, ist jedoch eine dadurch bedingte Fehlermöglichkeit äusserst gering.

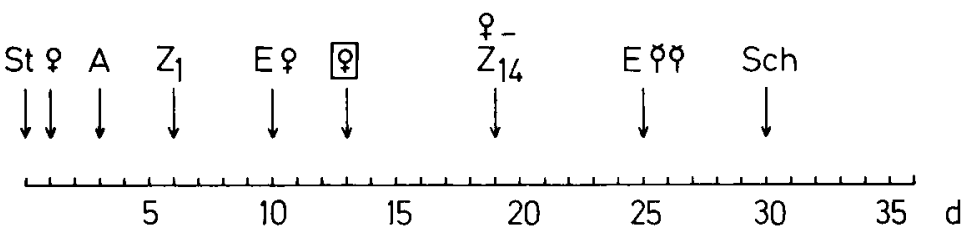

Aвв. 2. - Zeitliches Schema des Versuchsablaufes (in Tagen).

A. Aufstellung im Gelände; $q$ Schlüpfen der $q$ : 国 Käfigen der $q$;

Entnahme der $Q ;$ E $q$ Eiablage der $q ;$ E $\Varangle \zeta$ Eiablage von $\psi \not ;$

Sch Schlüpfen von Jungbienen ('Töchtei der $Q$ );

St Start mit Kunstschwarm; $Z_{1}$ Erstes Zusetzen markierter Jungbienen;

$\mathrm{Z}_{14}$ Letztes Zusetzen markierter Jungbienen.

Fig. 2. - Scheme of the experiment course.

A posting in the experimental field; $q$ emerging of the queen;

Q caging of the queen; $P$ - removal of the queen;

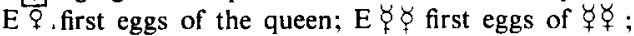

Sch emerging of young bees (daughters of the $q$ ); St start with artificial swarms;

$Z_{1}$ first young marked bees added; $Z_{14}$ last young marked bees added.

Aus technischen Gründen konnten zwar nicht alle Völkchen gleichzeitig angesetzt werden, aber immerhin lief der gesamte Versuch innerhalb der Hauptsaison des Bienenjahres ab (Tab. 2).

Im wesentlichen wurde der Versuch in zwei Gruppen durchgeführt: Die erste Gruppe mit 4 Herkünften zwischen Anfang Juni und Mitte Juli, die zweite Gruppe mit 3 Herkünften zwischen Mitte Juli und Anfang August.

Die Versuchsvölker wurden täglich auf das Vorhandensein von Eiern kontrolliert. Um zusätzliche quantitative Angaben über den Zustand der Ovarentwicklung zu gewinnen, wurden in regelmässigen Abständen Proben von 20 Bienen zur Präparation entnommen, beginnend am Tage der Entweiselung und abschliessend am Tage des Auftretens der ersten Eier. Jede Probe enthielt Bienen sämtlicher Altersstufen. Um den Arbeitsaufwand in vernünftigen Grenzen zu halten, wurde bei Festsetzung der Intervalle zwischen

TAB. 2. - Versuchszeitraum der untersuchten Rassen.

TABL. 2. - Experimental period of the races investigated.

\begin{tabular}{l|c|c}
\hline \multicolumn{1}{c|}{$\begin{array}{c}\text { Rasse } \\
\text { Race }\end{array}$} & $\begin{array}{c}\text { Beginn } \\
\text { Beginning }\end{array}$ & $\begin{array}{c}\text { Ende } \\
\text { End }\end{array}$ \\
\hline Ligustica (L) & 29.5. & 9.7. \\
Carnica (C) & 7.6. & 20.7. \\
Mellifera (M) & 7.6. & 13.7. \\
Adami (A) & 7.6. & 8.7. \\
Capensis (CAP) & 6.7. & 27.7. \\
Adansonii (AD) & 17.7. & 12.8. \\
Intermissa(IM) & 22.7. & 7.8. \\
\hline \hline
\end{tabular}


den Probenentnahmen die aus der Literatur und der eigenen Erfahrung zu erwartende Entwicklungsgeschwindigkeit der Ovarien berücksichtigt.

Diese Intervalle betrugen :

6 Tage für die Rassen Carnica, Ligustica, Mellifera und Adami.

3 Tage für das erste und 2 Tage für jedes weitere Intervall bei $A$ dansonii.

1 Tag bei Intermissa und Capensis.

Im grossen und ganzen entsprach der tatsächliche Verlauf der Ovarentwicklung diesen Erwartungen. So ergaben sich ziemlich gleichmässig 6-8 Proben je Volk. Lediglich bei der in ihrem Verhalten bisher weitgehend unbekannten $A$. m. adami waren es nur 4 Proben.

Die Bienenproben wurden in Weaver'schem Gemisch fixiert und später präpariert. Die Klassifizierung der Ovarien erfolgte nach VelthuIs, 1970, in 3 Stufen :

1. Unentwickelt.

2. Entwicklung stimuliert (Kammerung der Ovariolen).

3. Entwickelt mit morphologisch reifen Eiern.

Zur Klassifizierung wurde das jeweils stärker entwickelte der beiden Ovarien herangezogen. Je Volk und Entnahmedatum wurden 20 Bienen untersucht.

\section{RESULTATE}

\section{Einsetzen der Eiablage}

Der Zeitabstand zwischen der Entnahme der $q$ und dem Auftreten der ersten von $\Varangle \nvdash$ gelegten Eiern wird als Latenzzeit bezeichnet. Im allgemeinen standen 3 Völkchen zur Auswertung zur Verfügung. Bei $A . m$. adansonii waren es vier, bei A.m. capensis hingegen nur zwei Völkchen, da in zwei Völkchen die $q$ verschwunden war, ohne dass der genaue Zeitpunkt angegeben werden konnte.

\section{Tab. 3 gibt eine Übersicht über die Werte der Latenzzeit (in Tagen).}

Es ergeben sich deutlich drei Gruppen : Die europäischen Rassen Carnica Ligustica - Mellifera mit langer Latenzzeit, die afrikanischen Rassen Adansonii Intermissa-Capensis mit kurzer Latenzzeit und drittens $A$. $m$. adani, ein anatolisches

TAB. 3. - Latenzzeit (in Tagen) zwischen Entweiselung und Ablage der ersten Eier durch $\Varangle \zeta$ bei 7 Bienenrassen.

TABL. 3. - Latency period (days) between dequeening and laying of the first eggs by workers in 7 bee races.

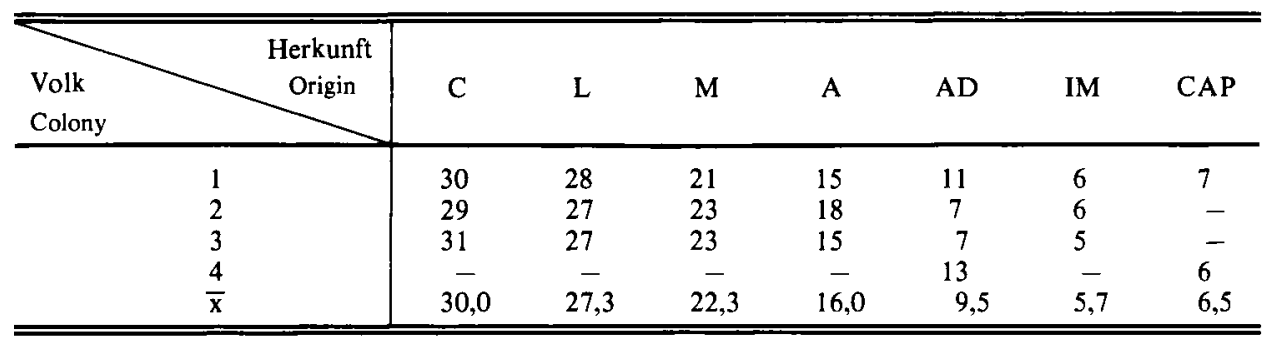


Faunenelement mit einem Zwischenwert (Abb. 3). Aber auch innerhalb der Gruppen zeigen die einzelnen Rassen deutlich verschiedene Werte der Latenzzeit, mit bemerkenswert geringer Streuung und nichttransgredierender Verteilung - abgesehen von den beiden afrikanischen Rassen Intermissa und Capensis mit besonders kurzer Latenzzeit.

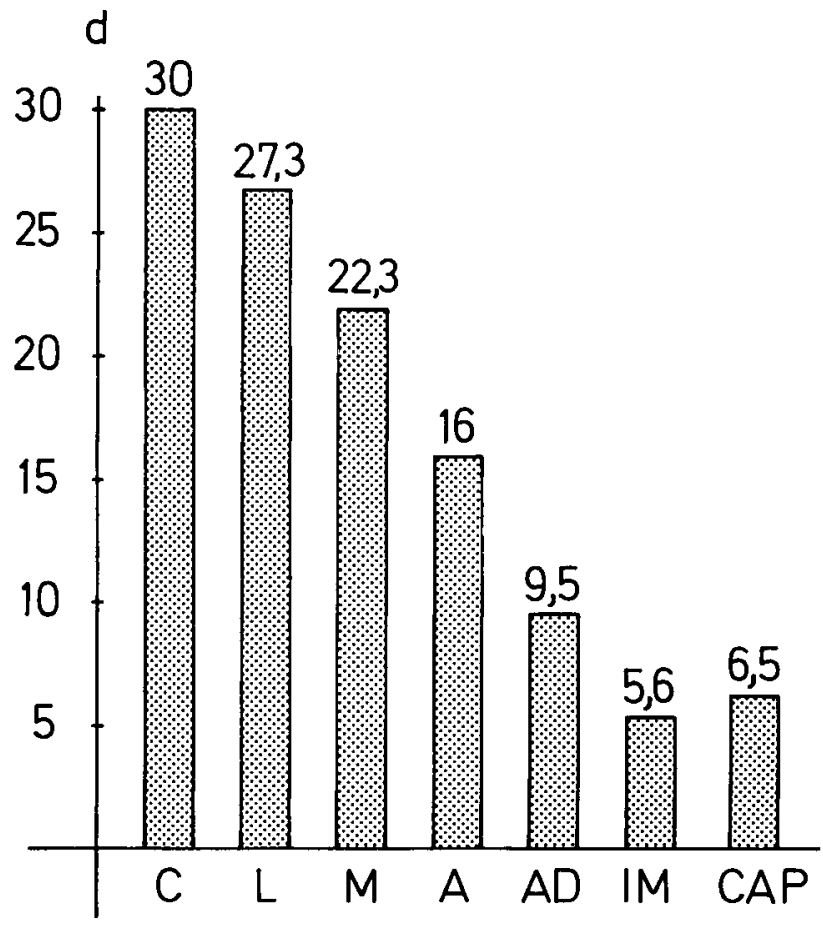

Aвв. 3. - Latenzzeit bei verschiedenen Bienenrassen (Mittelwerte in Tagen). C : Apis mellifera carnica; L : A. m. ligustica; M:A. m. mellifera;

A : A. m. adami; AD : A. m. adansonii-scutellata; IM : A. m. intermissa; CAP : A. m. capensis. FIG. 3. - Average latency period in different races of bees (days).

Statistisch lassen sich alle Werte auf dem $5 \%$-Niveau gegeneinander absichern mit Ausnahme derjenigen der Kapbiene gegenüber Adansonii und Intermissa (einseitiger U-Test nach WiLcoxon; Tab. 4).

\section{Anzahl der abgelegten Eier}

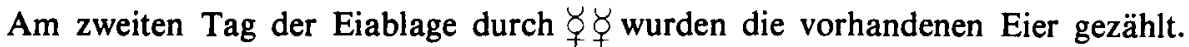
In den Völkchen war vorher durch die entnommene $q$ eine Wabe bebrütet worden, und

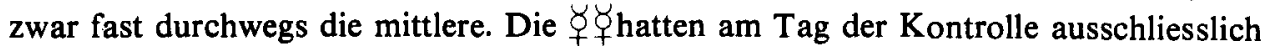
diese bebrütete Wabe zur Ablage von Eiern benutzt. 
TAB. 4. - Statistische Signifikanz der Unterschiede in der Latenzzeit ( $+\cdots: \mathrm{p}=0,05)$.

TABL. 4. - Statistical significance of differences in latency period $(\alpha+\cdots: p=0,05)$.

\begin{tabular}{|c|c|c|c|c|c|c|c|}
\hline & C & $\mathrm{L}$ & $\mathbf{M}$ & A & $\mathrm{AD}$ & IM & CAP \\
\hline $\begin{array}{l}\mathrm{C} \\
\mathrm{L} \\
\mathrm{M} \\
\mathrm{A} \\
\mathrm{AD} \\
\mathrm{IM} \\
\mathrm{CAP}\end{array}$ & & + & + & $\begin{array}{l}+ \\
+ \\
+\end{array}$ & $\begin{array}{l}+ \\
+ \\
+ \\
+\end{array}$ & $\begin{array}{l}+ \\
+ \\
+ \\
+ \\
+\end{array}$ & $\begin{array}{l}+ \\
+ \\
+ \\
+ \\
- \\
-\end{array}$ \\
\hline
\end{tabular}

Die mittlere Eizahl schwankt zwischen 27,7 bei $\mathrm{A} . \mathrm{m}$. mellifera und $655,3 \mathrm{~A} . \mathrm{m}$. adami (Tab. 5, Abb. 4). Alle drei europäischen Rassen weisen geringe Eizahlen auf, obwohl ja bekanntlich im Normalvolk die Italienische und die Kärntner Biene zu den stark brütenden Rassen gehören.

\section{Aвв. 4. - Mittlere Eizahl in den ersten 48 Stunden nach Beginn der Legetätigkeit der Arbeitsbienen. \\ Symbole der Rassen wie in Abb. 3.}

FIG. 4. - Average number of eggs laid during the first 48 hours of egg laying by workers.

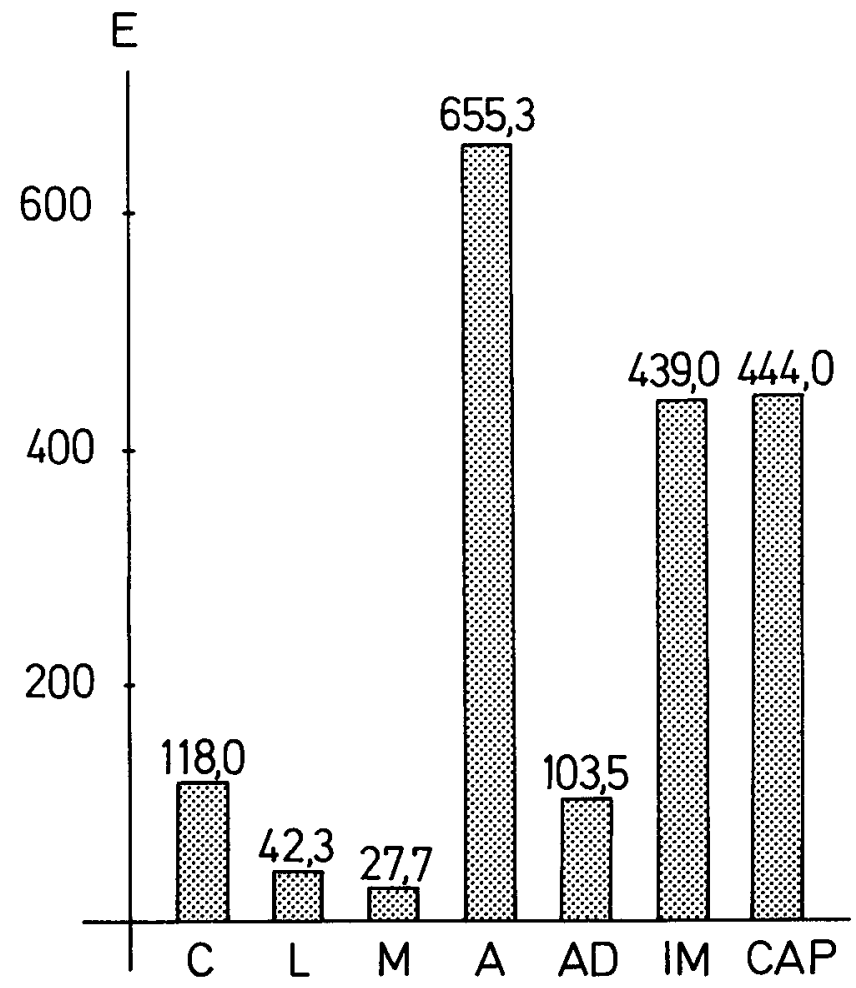


TAB. 5. - Anzahl der in den ersten 48 Stunden abgelegten Eier.

TABL. 5. - Number of eggs laid during the first 48 hours.

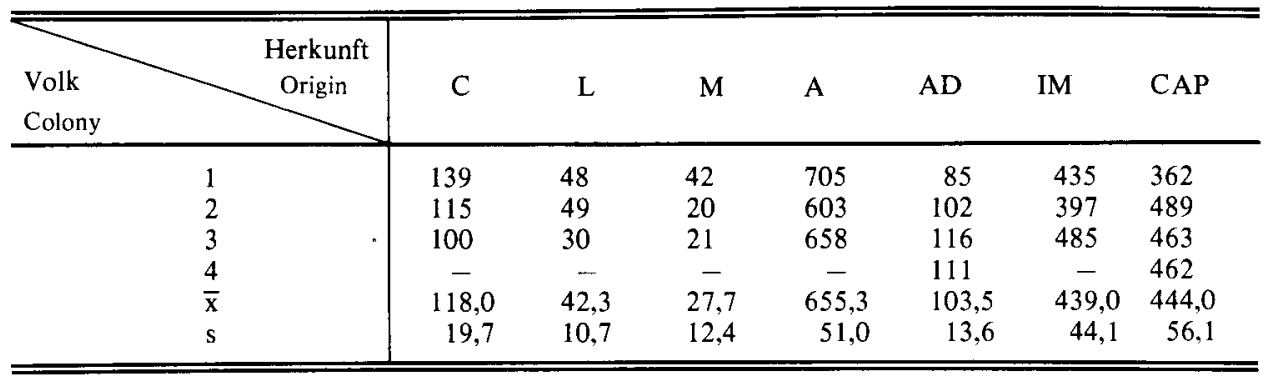

Es besteht eine Beziehung zwischen Latenzzeit und Zahl der abgelegten Eier (parameterfreier Rangkorrelationstest nach SPEARMAN $\mathbf{r}_{\mathbf{s}}=-0,507$, signifikant auf Niveau 0,025) : Bienen mit langer Latenzzeit legen im allgemeinen weniger Eier als solche mit kurzer.

Das Legepotential der $\not \Varangle q$ zu Beginn ihrer Reproduktionsphase scheint nach den vorliegenden Ergebnissen charakteristisch für die einzelnen Rassen zu sein. Wie bei der Latenzzeit ist auch hier die Streuung innerhalb der Gruppe gering. Bei paarweisem Vergleich der Rassen sind nur die drei Paare Ligustica-Mellifera, Carnica-Adansonii und Intermissa-Capensis gegeneinander nicht signifikant abzusichern (U-Test von WiLCoxon, $p=0,05$; Tab. 6).

Trägt man in einem Koordinatensystem Eizahl gegen Latenzzeit auf, so kommt man zu einer guten Trennung der Gruppen (abgesehen von intermissa-capensis; Abb. 4). Diese beiden Parameter können demnach möglicherweise als charakteristische Rassenmerkmale gewertet werden.

\section{Anzahl der Ovariolen pro Ovar}

Die Anzahl der abgelegten Eier könnte korreliert sein mit der Ovariolenzahl. Deshalb wurde an zwei zufällig entnommenen Stichproben je Herkunft die

TAB. 6. - Statistische Signifikanz der Unterschiede in der Eizahl.

TABL. 6. - Statistical significance of differences in egg number.

\begin{tabular}{|c|c|c|c|c|c|c|c|}
\hline & C & $\mathrm{L}$ & M & A & $\mathrm{AD}$ & IM & $\mathrm{CAP}$ \\
\hline $\mathrm{C}$ & & + & + & + & - & + & + \\
\hline L & & & - & + & + & + & + \\
\hline M & & & & + & + & + & + \\
\hline A & & & & & + & + & + \\
\hline $\mathrm{AD}$ & & & & & & + & + \\
\hline IM & & & & & & & - \\
\hline CAP & & & & & & & \\
\hline
\end{tabular}


Ovariolenzahl bestimmt. Da sich diese Zahl nur bei entwickelten Ovarien ohne Hilfsmittel (Einbettung, Färbung) exakt feststellen lässt, wurden nur Ovarien der Stufe 3 herangezogen.

Auch bei der Ovariolenzahl treten - ähnlich wie bei der Latenzzeit - drei unterscheidbare Gruppen hervor (Tab. 7) : Europa und Afrika als Extreme und der Vordere Orient in einer Mittelstellung. Erwartungsgemäss wurde bei Capensis die höchste Ovariolenzahl gefunden. Auch die Ovariolenzahl ist - mit gewissen Einschränkungen - ein rassenspezifisches Merkmal. Die europäischen Rassen sind untereinander nicht signifikant verschieden, dasselbe gilt für Adami gegenüber Adansonii und Intermissa wie Adansonii gegen Intermissa (Test von KOLMOGOROFF und SMIRNOFF; Tab. 8).

Die europäischen Rassen, mit der niedrigsten Eizahl, weisen auch die geringste Anzahl von Ovariolen auf. Trotzdem lässt sich keine allgemeine Korrelation zwischen diesen beiden Parametern ableiten. Das zeigt etwa der Vergleich zwischen den Rassen Adami - Adansonii - Intermissa: Die Zahl der Ovariolen liegt bei allen dreien in derselben Grössenordnung, die Eizahlen aber sind ganz verschieden. Ganz ähnlich ist das Verhältnis bei dem Vergleich Carnica-Mellifera : Die Ovariolenzahlen sind gleich, aber die Eizahl ist bei der Carnica viermal so gross.

Der Spearmansche Rangkorrelationskoeffizient $r_{s}=0,613$ ist nicht signifikant. Hingegen ergibt sich eine signifikante negative Korrelation zwischen Latenzzeit und

TAB. 7. - Anzahl der Ovariolen.

TABL. 7. - Number of ovarioles.

\begin{tabular}{|c|c|c|c|}
\hline $\begin{array}{c}\text { Rasse } \\
\text { Race }\end{array}$ & $\mathrm{n}$ & $\bar{x}$ & $\begin{array}{c}\text { Grenzwerte } \\
\text { extreme values }\end{array}$ \\
\hline $\begin{array}{l}\text { Carnica } \\
\text { Ligustica } \\
\text { Mellifera } \\
\text { Adami } \\
\text { Adansonii } \\
\text { Intermissa } \\
\text { Capensis }\end{array}$ & $\begin{array}{l}22 \\
22 \\
19 \\
24 \\
31 \\
20 \\
33\end{array}$ & $\begin{array}{l}3,26 \\
3,73 \\
3,21 \\
5,70 \\
7,1 \\
7,4 \\
9,4\end{array}$ & $\begin{array}{l}2-6 \\
2-6 \\
2-5 \\
3-9 \\
5-9 \\
5-10 \\
8-14\end{array}$ \\
\hline
\end{tabular}

TAB. 8 - Statistische Signifikanz der Unterschiede in der Ovariolenzahl.

TABL. 8. - Statistical significance of differences in ovariole number.

\begin{tabular}{|c|c|c|c|c|c|c|c|}
\hline & $\mathrm{C}$ & $\mathrm{L}$ & $\mathbf{M}$ & A & $\mathrm{AD}$ & IM & CAP \\
\hline $\begin{array}{l}\mathrm{C} \\
\mathrm{L} \\
\mathrm{M} \\
\mathrm{A} \\
\mathrm{AD} \\
\mathrm{IM} \\
\mathrm{CAP}\end{array}$ & & - & $\begin{array}{l}- \\
-\end{array}$ & $\begin{array}{l}+ \\
+ \\
+\end{array}$ & $\begin{array}{l}+ \\
+ \\
+ \\
--\end{array}$ & $\begin{array}{l}+ \\
+ \\
+ \\
- \\
-\end{array}$ & $\begin{array}{l}+ \\
+ \\
+ \\
+ \\
+ \\
+\end{array}$ \\
\hline
\end{tabular}




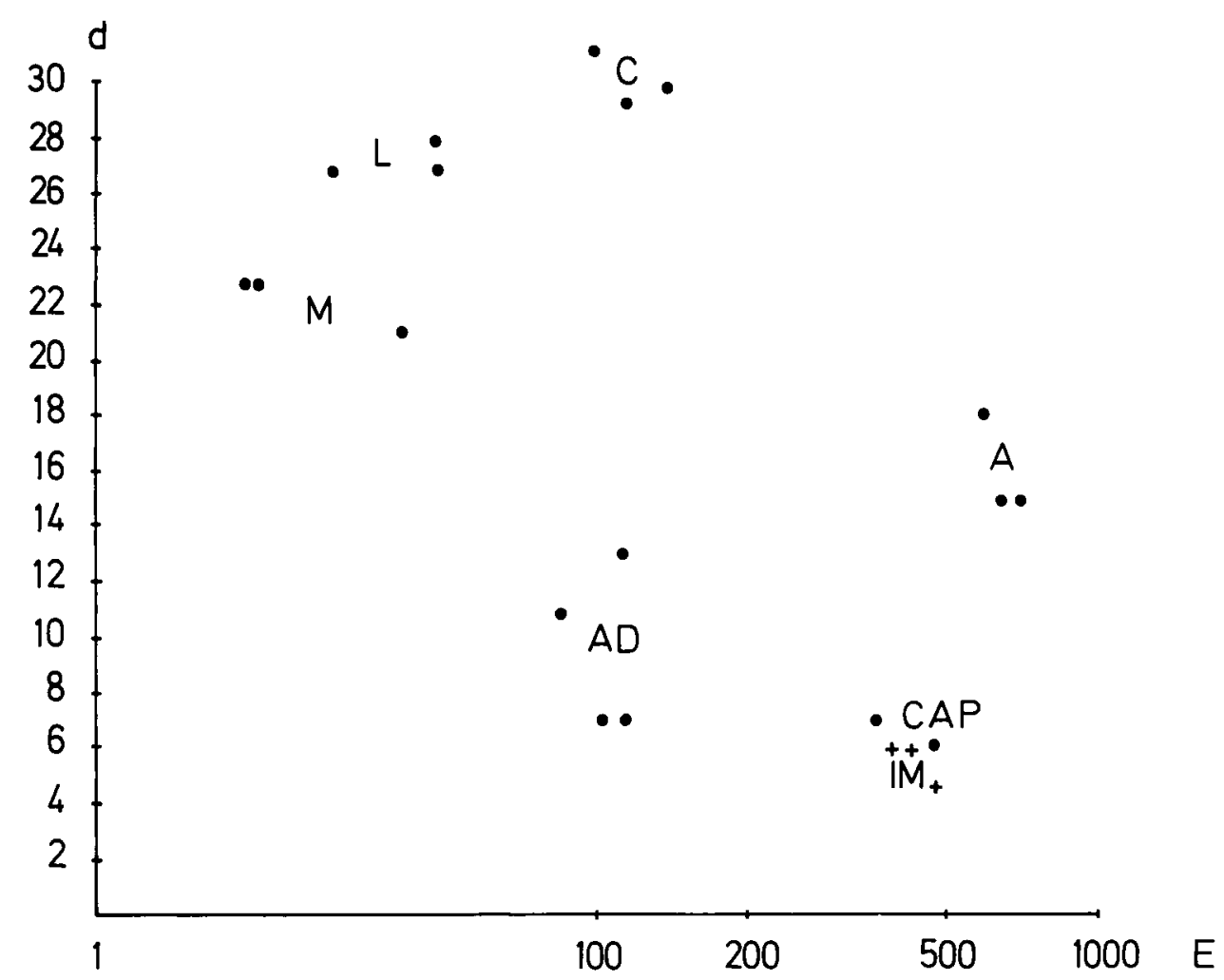

Aвв. 5. - Korrelation zwischen Eizahl (E) und Latenzzeit (d) in Tagen. Symbole der Rassen wie in Abb. 3.

FIG. 5. - Correlation between egg number $(E)$ and latency period (days $=\mathrm{d})$.

Ovariolenzahl $\left(r_{s}=-0,857\right)$. Je kürzer die Latenzzeit, desto grösser die Zahl der Ovariolen (Abb. 6).

Es besteht eine Beziehung zwischen Latenzzeit und Zahl der abgelegten Eier (parameterfreier Rangkorrelationstest nach Spearman $r_{s}=-0,507$, signifikant auf dem Niveau 0,025) : Bienen mit langer Latenzzeit legen im allgemeinen weniger Eier als solche mit kurzer.

\section{Entwicklungsgrad der Ovarien}

Im Ausgangsstadium, bei der ersten Probenentnahme am Tage der Entweiselung, waren die Ovarien bei sämtlichen Rassen so gut wie unentwickelt (Abb. 7). Stufe 3 wurde bei keiner einzigen Biene gefunden. Stufe 2 gab es zu diesem Zeitpunkt nur bei aussereuropäischen Rassen : Bei Adami $1 \times$ (unter 60 Bienen), bei Adansonii $4 \times$ (unter 80 Bienen $)$, bei Intermissa $1 \times(60$ Bienen $)$ und bei Capensis $2 \times(40$ Bienen $)$. 


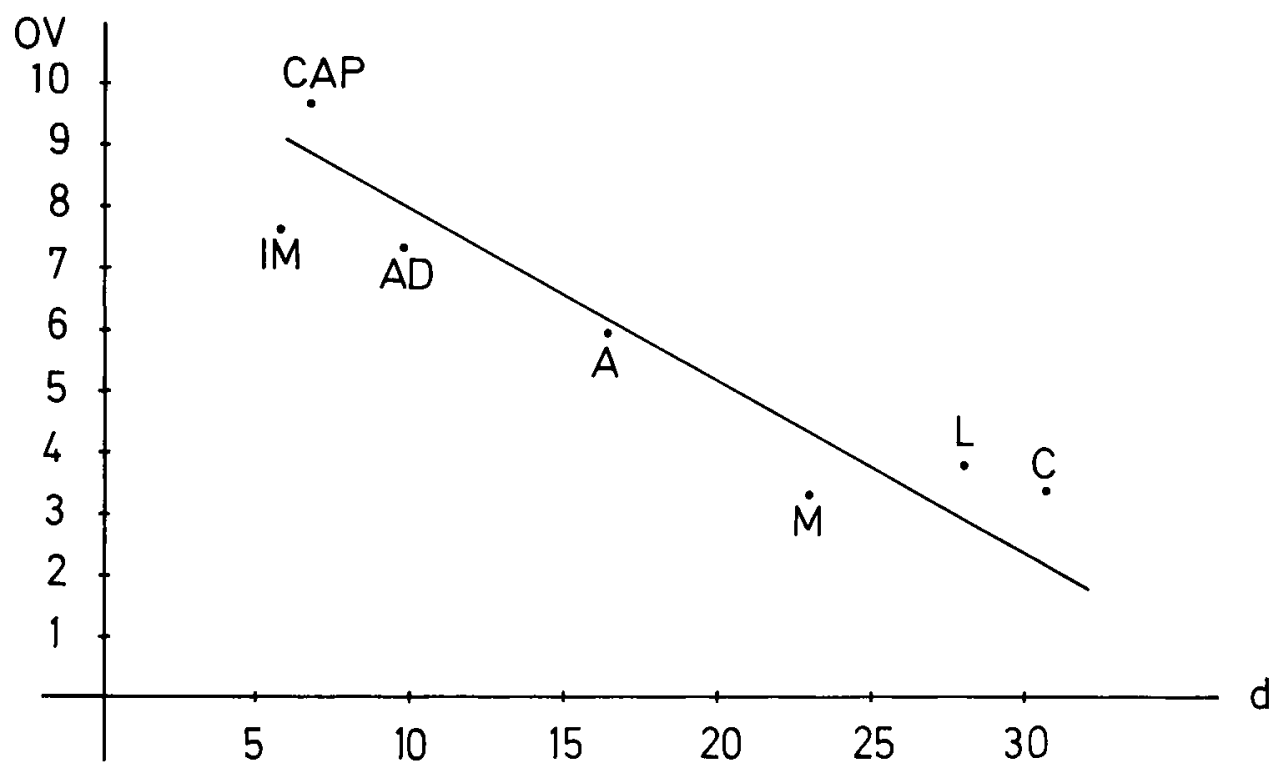

AвB. 6. - Latenzzeit (d) in Funktion der Ovariolenzahl (OV) Symbole der Rassen wie in Abb. 3.

FIG.6. - Latency period (d) against ovariole number (OV).

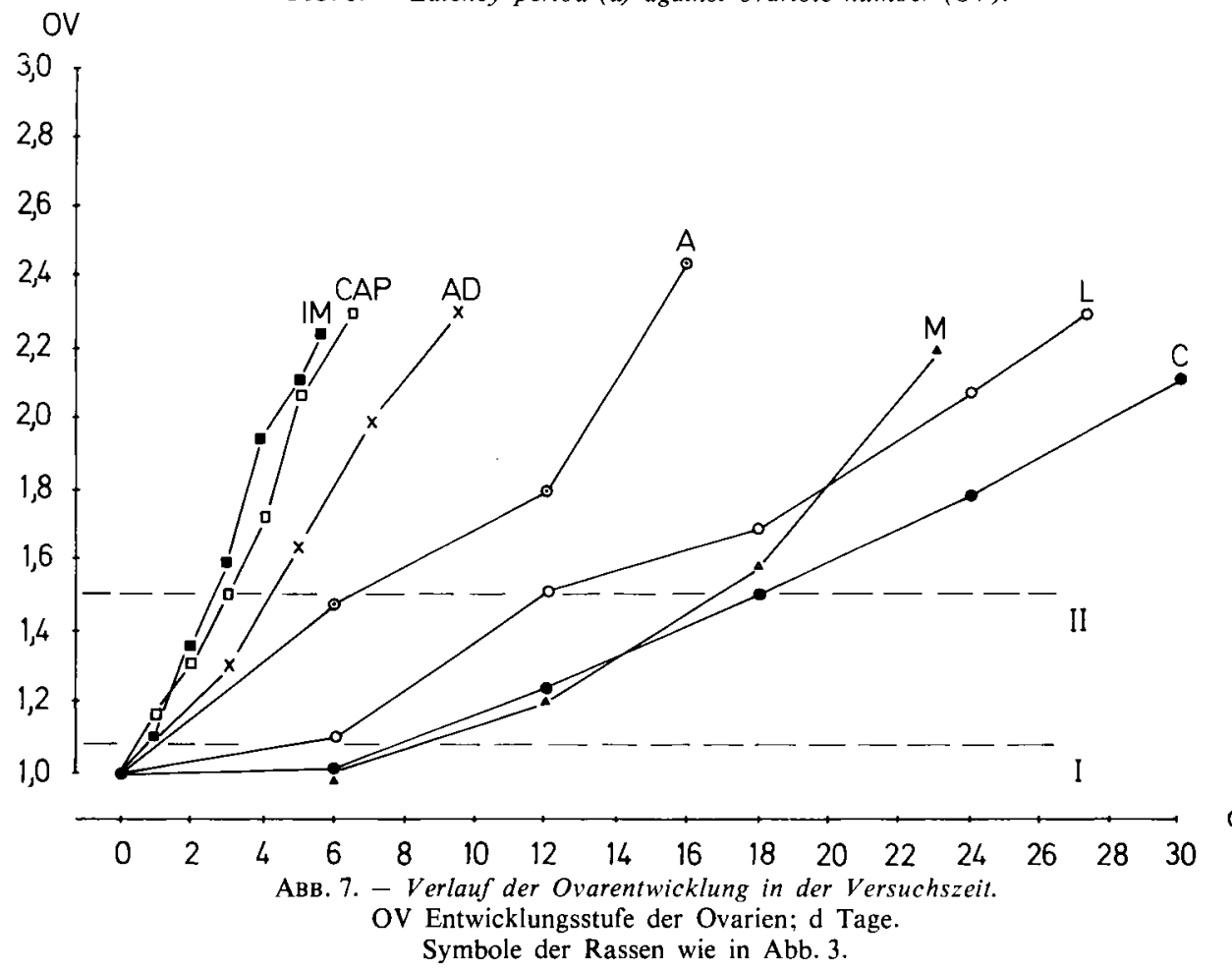

FIG. 7. - Course of ovary development during the experimental period. 
In der Abschlussprobe, entnommen zu Beginn der Eiablage, waren die Mittelwerte der Entwicklungsstufen für alle Rassen fast identisch (Tab. 9).

TABL. 9. - Mittlerer Entwicklungszustand

der Ovarien zum Zeitpunkt des Beginns der Eiablage.

ТАВ. 9. - A verage development stage of the ovaries on the day of start of egg laying.

\begin{tabular}{l|ccccccc}
\hline \multicolumn{1}{c|}{$\begin{array}{c}\text { Rasse } \\
\text { Race }\end{array}$} & C & L & M & A & AD & IM & CAP \\
\hline $\begin{array}{l}\text { Entwicklungsstufe d. Ovarien, } \bar{x} \\
\text { Ovaries, stage of development }\end{array}$ & 2,13 & 2,29 & 2,21 & 2,46 & 2,31 & 2,25 & 2,31 \\
\hline \hline
\end{tabular}

Offenbar ist der physiologische Zustand der Ovarien, der bei Einsetzen der Eiablage erreicht sein muss, bei allen Rassen gleich.

Von wenigen Ausnahmen abgesehen, tauchten die ersten Bienen mit voll entwickelten Ovarien fast ausschliesslich bei der vorletzten Kontrolluntersuchung auf (also bei der fünften der sechs Untersuchungen). Zwischen dieser und dem Auftreten der ersten Eier, also in den letzten Versuchstagen, steigt die Anzahl der Bienen mit den entwickelten Ovarien meist sprunghaft an - von 1 auf 3 oder von 2 auf 7.

Die Hemmwirkung der frühentwickelten Bienen auf die übrigen Mitglieder der Gruppe scheint demnach nicht gross zu sein. Das geht auch aus der Linearität des Anstieges hervor (s. unten). Offenbar ist diese Situation, in der sich alle Bienen der Gruppe ungefähr in derselben Ausgangsverfassung befunden haben, sehr verschieden von der Versuchsanordnung, in der eine Arbeiterin mit entwickelten Ovarien zII unentwickelten Versuchsbienen gesetzt wurde (Tab. 1).

Die Zahl der Bienen mit voll entwickelten Ovarien in der Gruppe von 20 schwankte zum Zeipunkt des Eilagebeginns zwischen 3 und 10 (15-50\%).

Es könnte sein, dass bei einigen Rassen einfach deshalb mehr Eier abgelegt werden als bei anderen, weil mehr legende $\not{\gamma} \not$ vorhanden sind. Bei $A d a m i$ trifft dies Offenbar zu : Sie hat die höchste Eizahl und die höchste Zahl Bienen mit entwickelten Ovarien (im Mittel 8,3 pro Käfig). Aber im Gesamten lässt sich keine allgemeine Regel aufstellen (Tab. 10).

Offensichtlich entstehen die grossen Unterschiede in der Legeleistung der einzelnen Rassen durch Zusammenwirken mehrerer Faktoren, wie der Zahl legender Bienen, der individuellen Legeleistung und vermutlich noch anderer.

Wie schon aus den sehr unterschiedlichen Latenzzeiten zu erwarten, ist hingegen der zeitliche Verlauf der Ovarentwicklung bei den untersuchten Rassen sehr verschieden. Bei gleicher Höhe der Anfangs- und Endpunkte zeigt der Winkel der Kurvenneigung sehr unterschiedliche Werte (Abb. 7).

Wie aus Abb. 7 hervorgeht, verläuft die Ovarentwicklung nach einer "Anlaufphase ", die bei europäischen Rassen bis zu 6 Tagen dauern kann, annähernd 
TABL. 10. - Mittlere Zahl der Bienen mit entwickelten Ovarien

(in einer Versuchsgruppe von 20 Bienen)

$z u$ Beginn der Eiablage (=funktionelle Legerinnen)

bei Völkern mit geringer, mittlerer und hoher Eizahl.

TAB. 10. - Average number of bees with developed ovaries

(in an experimental group of 20 bees)

at the beginning of egg laying (= functional layers)

in colonies with low, medium and high egg number.

\begin{tabular}{|c|c|c|c|c|c|c|c|c|c|}
\hline \multicolumn{3}{|c|}{$\begin{array}{c}\text { Eizahl }<50 \\
\text { Egg number }<50\end{array}$} & \multicolumn{3}{|c|}{$\begin{array}{c}\text { Eizahl } \simeq 100 \\
\text { Egg number } \simeq 100\end{array}$} & \multicolumn{4}{|c|}{$\begin{array}{c}\text { Eizahl }>400 \\
\text { Egg number }>400\end{array}$} \\
\hline $\mathbf{M}$ & $\mathbf{L}$ & $\overline{\mathbf{x}}$ & C & $\mathrm{AD}$ & $\overline{\mathrm{x}}$ & IM & A & CAP & $\overline{\mathbf{x}}$ \\
\hline 5,7 & 4,7 & 5,2 & 4,0 & 6,0 & 5,0 & 4,7 & 8,3 & 6,3 & 6,4 \\
\hline
\end{tabular}

linear. Nur bei Capensis setzt die Ovarentwicklung beinahe unmittelbar nach der Entweiselung, innerhalb der ersten 24 Stunden, ein. Die Untersuchung des Kurvenverlaufs mittels der Trendprüfung nach YATES (1948) ergibt, dass die Häufigkeiten in Abhängigkeit einer Merkmalsreihe linear zunehmen. Dies ist bei allen Rassen auf dem 0,01 Niveau signifikant. Irgendein Einfluss eines neu hinzukommenden Faktors im Verlaufe der Ovarentwicklung - etwa die Hemmwirkung von $\not \Varangle \ngtr$ mit schon stärker entwickelten Ovarien - lässt sich also nicht nachweisen.

\section{DISKUSSION}

Wie in der Einleitung erwähnt, liegen sehr viele Daten darüber vor, dass die Entwicklung der Ovarien von $\not{q} \not{+}$ von einer ganzen Anzahl von Umweltfaktoren beeinflusst wird : Versorgung mit Eiweiss, Jahreszeit, physiologischer Zustand des

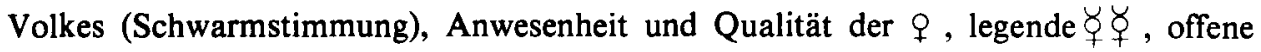
Brut. Für den Nachweis zusätzlicher genetischer Faktoren musste daher einer

ТАВ. 11. - Entwicklungsgeschwindigkeit der Ovarien bei älteren und bei jüngeren Arbeiterinnen.

(+ : Entwicklungsgrad der Ovarien bei jüngeren Arbeiterinnen signifikant höher.)

TABL. 11. - Rapidity of ovary development in elder and younger workers.

(" + " : development of ovaries significantly higher in younger workers.)

\begin{tabular}{|c|c|c|c|c|c|c|c|c|}
\hline & 1 & 2 & 3 & 4 & 5 & 6 & 7 & 8 \\
\hline $\mathrm{C}$ & - & - & + & + & + & + & & \\
\hline L & - & + & + & + & - & - & & \\
\hline $\mathbf{M}$ & - & - & - & - & + & & & \\
\hline A & - & - & - & - & & & & \\
\hline $\mathrm{AD}$ & - & - & - & - & - & - & - & + \\
\hline IM & - & - & - & - & - & + & + & \\
\hline CAP & - & - & - & - & - & - & - & + \\
\hline
\end{tabular}


möglichst sorgfältig kontrollierten Versuchsmethodik eine zentrale Bedeutung zukommen. Nur bei weitgehend gleichartigen und zugleich optimalen Umweltverhältnissen konnten aussagekräftige Ergebnisse erzielt werden. Die Reproduktion im Bienenvolk ist ein von der sozialen Gesamtstruktur gesteuertes Phänomen. Diese Struktur, bestimmt durch den inneren Aufbau des Volkes Alterszusammensetzung, Brut, Königin - und durch die äusseren Bedingungen, befindet sich in einem labilen Zustand, der durch experimentelle Eingriffe leicht gestört werden kann.

Bei der Planung der Experimente über die Ovarentwicklung der $\not \Varangle q$ musste ein Weg gefunden werden, die Versuchsbienen vorsichtig in ein normal aufgebautes, vitales Volk einzuschleusen und ihre Entwicklung unter optimalen, weitgehend störungsfreien Bedingungen $\mathrm{zu}$ verfolgen.

Diese Grundforderungen wurden in der gewählten Methodik so weit als möglich eingehalten. Es wurden Kunstschwärme mit Bienen aller Altersstufen gebildet, mit einer $q$, die inmitten ihrer Bienen ausgeschlüpft war. Die Paarungen der $q q$ erfolgten rasch und mit sehr geringen Ausfällen. So standen vollaktive kleine Völker mit jungen, legenden $\$ q$ zur Verfügung, die nun täglich eine Gruppe (80-200) frischgeschlüpfer, markierter $\not \zeta$ erhielten. Diese Versuchsbienen reiften also in einem weiselrichtigen Volk in gutem Ernährungszustand und mit offener Brut heran. Zur Vorbereitung des Versuches wurde die $q$ im Volk gesperrt, um für den richtigen Zeitpunkt den erforderlichen brutfreien Zustand zu erreichen. Zum Zeitpunkt der Entweiselung des Volkes waren nur ältere Larven und drei Tage später ausschliesslich verdeckelte Brut vorhanden, die zwar das weisellose Volk stabilisierte, aber auf die Ovarentwicklung der $\not \gamma$ keinen Einfluss hatte. Die Pflegebienen des "Trägervolkes » wurden zusammen mit der $q$ entnommen; das Versuchsvolk bestand jetzt ausschliesslich aus datierten Bienen der zu untersuchenden Rasse, dessen Altersaufbau (0-15 Tage) im Bereich der physiologischen Regulationsfähigkeit auch für die Sammeltätigkeit lag (LINDAUER, 1952) und die in einem weiselrichtigen, aktiven Volk herangereift waren.

Als Einwand könnte vorgebracht werden, dass die Versuche nicht alle zu exakt derselben Zeit durchgeführt werden konnten. Dazu ist jedoch $z \mathfrak{u}$ sagen, dass der gesamte Versuchszeitraum (Anfang Juni - Anfang August) in die " hohe Zeit " der Bienensaison fällt. Es ist derselbe Zeitabschnitt, in dem VelTHuIs (1970) den Höhepunkt der Ovarentwicklung fand (Mitte Juni-Mitte August). Erst im Herbst erfolgt die Entwicklung langsamer (Hess, 1942). Als Folge der unterschiedlichen Verfügbarkeit des Materials ergab es sich, dass die Versuche mit Rassen mit langsamer Ovarentwicklung in der ersten Hälfte des Versuchszeitraumes, die Versuche mit Rassen mit rascher Ovarentwicklung in der zweiten Hälfte durchgeführt wurden. Ein etwaiger Saisoneinfluss müsste sich also auf genetische Unterschiede nivellierend und nicht verstärkend auswirken. 
Dass der Versuch unter biologisch adäquaten Bedingungen durchgeführt wurde, geht auch daraus hervor, dass die Völkchen trotz Weisellosigkeit bis zum Abschluss des Experimentes in guter Verfassung blieben. Nur bei der Rasse Mellifera, mit relativ langer Latenzzeit, war gegen Ende ein deutliches Absinken der Populationsstärke zu verzeichnen.

Demnach können die festgestellten Unterschiede in der Entwicklungsgeschwindigkeit der Ovarien und in der Eilegerate mit gutem Grund als endogen verursacht bewertet werden. Dafür spricht auch die geringe Streuung der Werte innerhalb der Gruppe.

Als Ergebnis des Versuches ist festzuhalten, dass jede Rasse ihre spezifische Latenzzeit (=Zeitspanne zwischen Entnahme der $q$ und Beginn der Eiablage durch die $\not \Varangle \not$ ) hat. Es lassen sich drei Gruppen unterscheiden : Die europaïschen Rassen mit 3-4 Wochen Latenzzeit, die der ostmediterranen Rassengruppe zugehörige A. $m$. adami mit 2 Wochen und schliesslich die afrikanischen Rassen mit einer Woche Latenzzeit und darunter. Aber auch die einzelnen Rassen innerhalb der Gruppe weisen signifikant verschiedene Werte auf, abgesehen von den beiden afrikanischen Rassen A. m. intermissa und adansonii gegenüber A.m. capensis, die alle eine sehr kurze Latenzzeit haben. Möglicherweise könnte aber auch innerhalb dieser Gruppe mit grösseren Stichproben eine bessere statistische Abgrenzung gelingen.

Eine kurze Latenzzeit ist die Folge einer raschen Entwicklung der Ovarien nach Entfernung der $q$, also eines steilen Ansteigens der Regressionsgeraden Entwicklungsstufe/Zeit (Abb.7). Ausgangspunkt dieser Kurven war bei fast allen Völkern der Wert 1, d.h. die Bienen hatten in den weiselrichtigen Völkern unentwickelte Ovarien. Zum Zeitpunkt des Auftretens der ersten Eier war der mittlere Entwicklungsgrad der Ovarien bei allen Gruppen annähernd gleich, ungeachtet des unterschiedlichen Zeitraumes (zwischen 5 und 31 Tagen).

Mit Werten von 2,2-2,4 ist der Entwicklungsgrad zu diesem Zeitpunkt relativ hoch, die Mehrzahl der Bienen hat stimulierte Ovarien und $15-50 \%$ sogar voll entwickelte Ovarien (Stufe 3). Das zeigt, dass in diesen Völkchen - zunächst zumindest - eine allgemeine Ovarentwicklung eingesetzt hat und dass die Hemmwirkung der $\Varangle \Varangle$ mit voll entwickelten Ovarien auf die übrigen Bienen nicht sehr stark sein konnte. Das war der Befund beim Auftreten der ersten Eier. Ob sich in der Folge der Einfluss der legenden $\Varangle \Varangle$ stärker bemerkbar gemacht hat, wurde nicht untersucht. Nach den Befunden bei Capensis haben legende $\Varangle \not q$ bei längerem Einwirken eine ähnliche Hemmwirkung wie $q q$ (s. S. 4).

Bei $A$ pis cerana und $A$. florea sind nach noch unveröffentlichten Ergebnissen aus diesem Institut beim Auftreten legender $\not \Varangle \zeta$ die Ovarien bei einer weit geringeren Zahl der Bienen entwickelt, d.h. der allgemeine Entwicklungsgrad ist geringer. Offensichtlich handelt es sich hier um artspezifische Unterschiede in der Hemmwirkung legender $\not \Varangle q$. 
Bei der Beurteilung der Befunde war zu überlegen, ob nicht das Alter der betreffenden $\not \Varangle \zeta$ einen Einfluss auf den Entwicklungsgrad der Ovarien hat. Um das zu überprüfen, wurden aus jeder Probe die Entwicklungsstufen der drei jüngsten und der drei ältesten Altersgruppen (am Tag der Entweiselung 0-3 Tage, bzw. 12-15 Tage alt) miteinander verglichen. Infolge der Unterschiede der Latenzzeit waren am Tage der letzten Probe (Zeitpunkt der Eiablage) bei der Carnica die jüngsten Bienen 30-33 und die ältesten Bienen 44-47 Tage alt, bei der Intermissa jedoch nur 6-8 Tage, bzw. 19-22 Tage.

Wie aus Tab. 11 ersichtlich, ergab sich nur bei der Carnica eine signifikant schnellere Ovarentwicklung bei Jungbienen als bei Altbienen (4-Feldertest, $p=5 \%$ ), wie dies JAY (1968) bei seinen Versuchsbienen gefunden hatte. Bei vier Rassen (Mellifera, Adansonii, Intermissa, Capensis) ergab sich nur bei der letzten Probe ein Unterschied, bei Adami Zu keinem Zeitpunkt. Es scheint somit nur bei den ältesten Bienen eine gewisse Hemmung der Ovarentwicklung aufzutreten.

Die in diesen Versuchen ermittelten Werte der Latenzzeit stehen in Übereinstimmung mit den imkerlichen Erfahrungen mit einigen Rassen, so hinsichtlich der europäischen Rassen durch v. BERLEPSCH (1873) und GONTARSKI (1938), von Capensis durch ONIONS (1912), ANDERSON (1960) und RUTTNER (1975), von Adansonii durch Fletcher (mündl. Mitteilung 1977) und von Intermissa (eigene Beobachtung). Das weist darauf hin, dass die in den Versuchen verwendeten Stichproben als repräsentativ für den Rassentyp gelten können und dass es sich bei der Latenzzeit, bzw. der Entwicklungsgeschwindigkeit der Ovarien um ein genetisch fixiertes Merkmal handelt, dass auch unter abweichenden Klima- und Trachtverhältnissen nicht grundlegend verändert wird.

Ein Widerspruch besteht hingegen bezüglich der Angaben von JAY (1975), der nach 10 Tagen Weisellosigkeit keinen Unterschied in der Ovarentwicklung zwischen afrikanischen und europäischen Rassen feststellen konnte. Da sich bei unseren Versuchen mit Originalstämmen aus dem Herkunftsland zu diesem Zeitpunkt alle drei afrikanischen Rassen schon voll in Eilage befanden, muss die Frage nach einer eventuellen Hybridisierung der von $\mathrm{J}_{\mathrm{AY}}$ verwendeten Bienen gestellt werden.

Voraussetzung für die Ovarentwicklung ist die vermehrte Produktion von Dotterproteinen, die ihrerseits gesteuert wird durch das endokrine System. ENGELS (1974) und ENGELS u. Fahrenhorst (1974) konnten festellen, dass sich bei $\not \Varangle$ im weiselrichtigen Volk nur bis zu ihrer Funktion als Ammenbienen ein höherer Vitellogenintiter aufbaut. Vom 5. Lebenstag an sinkt er wieder ab. Bei legenden $\Varangle \nvdash$ (Carnica) hingegen bleibt er auf einem hohen Niveau stehen; er ist bei dieser Biene nicht viel niedriger als bei einer legenden $P$.

Physiologisch können die Unterschiede in der Latenzzeit als Unterschiede in der endogenen Hemmung der potentiellen Reproduktionsfähigkeit der $\Varangle \Varangle$ gedeutet werden. "Langsame" Rassen mit hoher Latenzzeit, sind genetisch in ihrer Reproduktionsfähigkeit so stark gebremst, dass nach Wegfall aller äusseren 
Hemmfaktoren (Königin, Larven) das ganze System nur sehr zögernd und wenig ergiebig in Gang kommt. Eine Carnica-Stockbiene, im Alter von 10 Tagen weisellos geworden, wird erst im Greisenalter, mit 40 Tagen ihre ersten Eier ablegen.

Bei einigen afrikanischen Rassen hingegen, wie besonders bei Capensis und Intermissa, scheinen nur die exogenen Hemmfaktoren wirksam zu sein. Fallen diese weg, so starten unmittelbar, innerhalb von 24 Stunden, die Reproduktionsvorgänge. Dazu kommt, dass bei den " langsamen " Rassen die Eiablage nicht nur später beginnt, sondern dass sie i. allg. auch wesentlich schwächer ist - also eine verminderte Reproduktionsfähigkeit im ganzen vorliegt.

Als drittes rassenspezifisches Merkmal aus dem Bereich der Reproduktion von $\Varangle \nvdash$, neben der Latenzzeit (und Entwicklungsgeschwindigkeit der Ovarien) und der Eizahl, kann nach dieser Untersuchung die Zahl der Ovariolen angenommen werden.

Es treten wieder die drei schon erwähnten Gruppen mit deutlich verschiedenen Werten hervor, ausserdem lassen sich, abgesehen von den europäischen, die meisten Rassen auch einzeln voneinander abgrenzen. Auch darin kann man den Effekt von endogenen Hemmfaktoren während der Larvenentwicklung sehen.

J. Chaud-Netto und O.C. Bueno (1979) kommen in einer sehr eingehenden Untersuchung an Arbeiterinnen von A.m. "adansonii" und A.m. "ligustica" ebenfalls zu dem Ergebnis, dass die festgestellten Unterschiede zumindest zum Teil auf genetischen Faktoren beruhen. Nur sind die ermittelten Ovariolenzahlen von den hier mitgeteilten stark verschieden : In allen Gruppen hatte " Ligustica " mehr Ovariolen als "Adansonii ". Dazu ist zu bemerken, dass von den genannten Autoren der Rassenbegriff anders verwendet wird; ihre A. m. " adansonii " ist eine "Afrikanisierte " Biene aus Südamerika, also unkontrollierte Hybriden zwischen Importen aus Südafrika und europäischen Bienen; für A. m. "ligustica " wird die Herkunft nicht angegeben, vermutlich handelt es sich um einen der kommerziellen Stämme mit gelbem Farbmuster am Abdomen. Bei unseren eigenen Stämmen hingegen handelt es sich um Originalherkünfte von geographischen Rassen im zoologischen Sinne.

Die Vermutung liegt nahe, es könnte eine Korrelation bestehen zwischen der Eizahl und der Zahl der Ovariolen. Das ist aber nur in sehr beschränktem Masse der Fall. Die sehr starke Erhöhung der Eizahl bei einzelnen Rassen wird man daher weniger auf eine Erhöhung der Legeleistung der einzelnen Biene, als auf die Erhöhung

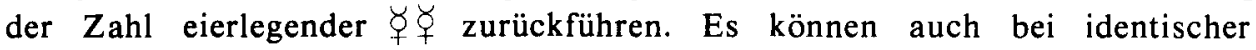
Ovariolenzahl sehr beträchtliche Unterschiede in der Zahl der abgelegten Eier auftreten : Wie SAKAGAMI et al. (1963) mitgeteilt haben, besitzen bei Melipona sowohl $q \uparrow$ wie $\not{q} \not{q}$ dieselbe Zahl von 4 Ovariolen. Experimentelle Daten über Unterschiede der Legeleistung von $q \subsetneq$ mit verschiedener Ovariolenzahl liegen für Apis jedoch nicht vor.

Hingegen besteht eine signifikante Beziehung zwischen Latenzzeit und Eizahl beides Ausdruck des vorhandenen Reproduktionspotentials (Abb. 5). 
Es macht einige Schwierigkeiten, sich eine Vorstellung vom Anpassungswert der Fortpflanzungsfähigkeit der $\nvdash \zeta z u$ bilden. Wie schon eingangs erwähnt, sind die Fortpflanzungsfunktionen der $\Varangle \Varangle$ an keiner Stelle des ungestörten Lebenszyklus des Bienenvolkes von essentieller Bedeutung. Vergleicht man auf einer Skala des Reproduktionspotentials die Werte der nordafrikanischen Tellbiene (Intermissa) mit einer der europäischen Rassen, so findet man letztere sehr weit unten, nahe dem Nullpunkt. Noch ein wenig Mehr an genetischer Hemmung und es wäre bei diesen Rassen die Fähigkeit zur Eiablage bei den $\Varangle \zeta$ zur Gänze erloschen. Dieser letzte Schritt ist jedoch bei keiner einzigen der bisher beobachteten Rassen erfolgt. Es ist also kaum zulässig, diesem Merkmal vorschnell jede adaptive Bedeutung abzusprechen.

Einen Sonderfall stellt die Kap-Biene dar. Sie besitzt die eigenartige und vielzitierte Fähigkeit, durch thelytoke Parthenogenese aus Eiern, die von $\not{q} \not$ stammen, wieder $\lceil\uparrow$ zu ziehen. Damit können weisellose Völker mit legenden $\nvdash \not$, die bei anderen Rassen dem Untergang verfallen sind, wieder in den Normalzustand zurückkehren. Voraussetzung dazu ist eine kurze Latenzzeit, damit der gaze Vorgang der $q q$ Aufzucht, Paarung und der Aufzucht neuer Brut trotz des fehlenden Nachschubs an Jungbienen bei guter Volksverfassung vor sich gehen kann. Es wurde die Hypothese diskutiert, dass unter dem Selektionsdruck starker $\Varangle \Varangle-V e r l u s t e$ aus klimatischen Gründen (auf der starken Winden ausgesetzten Kap-Halbinsel) in der Evolution ein solches Merkmal entstanden sein könnte (RUTTNER, 1976).

Schwieriger ist die Entwicklung von Vorstellungen über den adaptiven Wert der Fortpflanzung von $\not{\zeta} \not$ bei anderen Rassen, bei denen es fast ausschliesslich nur arrhenotoke Parthenogenese gibt. Völker mit legenden $\Varangle \zeta$ gehen - von seltenen Ausnahmefällen abgesehen - ein und die von ihnen erzeugten $\sigma^{x} \sigma^{x}$ erscheinen gewöhnlich erst nach Abschluss der allgemeinen Reproduktionsphase im Bienenvolk. Wenn eine Carnica- $\not{\succ}$ zur Zeit des Beginns der Eiablage 40 Tage alt ist - wie oben dargelegt - , dann hat sie bereits die durchschnittliche Lebensdauer einer Sommerbiene überschritten. Nun dauert es bis zur Erreichung der Geschlechtsreife der aus den Eiern entstehenden $\sigma^{7} \sigma^{7}$ noch einmal 36 Tage, also bis zum 76. Lebenstag dieser Bienen und wohl auch ihrer Stockgefährtinnen.

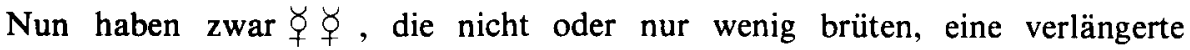
Lebensdauer (MAURIZIO, 1944), aber erfahrungsgemäss schwinden solche " buckelbrütigen "Völker dann doch sehr rasch dahin - zumal die $\sigma^{7} \sigma^{*}$ auch hohe Anforderungen an die Futterversorgung stellen. Die Funktionsdauer dieser $\sigma^{x} \sigma^{x}$ wird daher begrenzt sein. Dennoch gibt es eine Periode im Jahreszyklus des Bienenvolkes, in der sie unter bestimmten Umständen eine nicht unwesentliche Rolle spielen können.

Ein Bienenvolk in Normalverfassung erzeugt und pflegt $\sigma^{7} \sigma^{7}$ nur in Zeiten des Überflusses, also zur Vermehrungs- und Schwarmzeit. Geht das Nektarangebot zu Ende - und das kann in vielen Gegenden schon bald nach der Sommersonnenwende der Fall sein - dann werden die $\sigma^{x} \sigma^{x}$ sehr schnell und vollständig aus dem Volk vertrieben. 
Es tritt aber gar nicht selten der Fall ein, dass Bienenvölker noch spät im Jahr ihre alte $q$ durch eine junge ersetzen und dass noch im Herbst Hochzeitsflüge stattfinden. Um diese Zeit sind in weiselrichtigen Völkern keine $\sigma^{x} \sigma^{x}$ mehr vorhanden, wohl aber in buckelbrütigen - die ja nichts anderes erzeugen können als $\sigma^{7} \sigma^{7}$. Die regelmässige Erfahrung erfolgreicher Paarungen im Herbst beweist das Vorhandensein einer ausreichenden Drohnenpopulation - obwohl in den gut gepflegten Völkern des eigenen Standes $\sigma^{x} \sigma^{x}$ nicht mehr zu finden sind. Die einzige Quelle für $\sigma^{x} \sigma^{x}$ sind um diese Zeit weisellose Völker auf ungepflegten Bienenständen.

Regelmässige Erfahrungen auf Landbelegstellen stützen diese These : Die Quote von Reinpaarungen ist in der ersten Hälfte der Saison immer höher als an deren Ende, etwa im August. Um diese Zeit nimmt die Drohnenpopulation in den Belegstellenvölkern trotz sorgfältiger Pflege ab, so dass offensichtlich $\sigma^{x} \sigma^{7}$ aus der Umgebung (aus weisellosen Völkern) das Übergewicht erhalten.

So wird es vorkommen, dass in einer sich selbst überlassenen Bienenpopulation einzelne Völker nur dank der von $\not \Varangle \not$ stammenden $\sigma^{7} \sigma^{7} \mathrm{zu}$ einer begatteten $q$ kommen und überleben. Dieser Vorgang wird in heissen, extremen Klimagebieten eine grössere Rolle spielen als in Europa, weil dort die Überlebensstrategie der Bienen auf eine überschiessende Vermehrung gerichtet ist : Während in Trockenjahren $80 \%$ der Population zugrunde gehen können, werden in nachfolgenden guten Jahren die Verluste durch exzessives Schwärmen sehr rasch wieder wettgemacht. Über welch fast unglaubliche Vermehrungsrate afrikanische Bienen verfügen, haben sie nach ihrer Verpflanzung nach Südamerika gezeigt. Bei dieser hohen Fortpflanzungsrate - im gemässigten Klimabereich unbekannt - werden die aus weisellosen Völkern und Schwärmen stammenden $\sigma^{\star} \sigma^{\star}$ eine grössere Rolle spielen als bei Rassen mit geringer Schwarmtendenz. Dies könnte eine Erklärung anbieten für die Unterschiede in Latenzzeit und Eizahl zwischen afrikanischen und europäischen Rassen.

Eingegangen im März 1981

Reçu pour publication en mars 1981

\section{DANKSAGUNG}

Diese Arbeit wurde gefördert durch die Deutsche Forschungsgemeinschaft.

Für Unterstützung bei der statistischen Auswertung der Daten danken wir Robin MoRITz, für die Anfertigung der Graphiken Agnes MoHr. Bei der Beschaffung der Bienenstämme waren uns die Station expérimentale d'apiculture, Montfavet, die Station apicole Dr. SCHRöDER in Bir Bou Rekba (Tunesien) und Herr P. HÄssLER, Skeerport, Transvaal, behilflich.

\section{RÉSUMÉ}

DIFFÉRENCES RACIALES DANS LE DÉVELOPPEMENT OVARIEN ET LA PONTE DES OUVRIÈRES ORPHELINES D'APIS MELLIFERA L.

Pour déterminer le potentiel reproducteur de l'abeille ouvrière on a étudié les paramètres suivants sur des colonies en conditions naturelles : nombre d'ovarioles, déroulement du développement ovarien, début 
de la ponte (période de latence), œufs pondus pendant les premières 48 heures. Cette étude a porté sur les races suivantes : Apis mellifica carnica $(\mathrm{C})$, ligustica $(\mathrm{L})$, mellifera $(\mathrm{M})$, adami $(\mathrm{A})$, adansonii $(=$ scutella) (AD), capensis (CAP), intermissa (IM).

Les ouvrières écloses en éiuve sont marquées et introduites dans de petites colonies carnoliennes possédant une reine. Celle-ci est ensuite encagée et au bout de 5 jours retirée de la colonie avec les ouvrières éleveuses. Il reste alors une colonie expérimentale composée d'environ 1500 abeilles marquées d'une race donnée appartenant aux classes d'âge de 1 à 15 jours avec, au début, des larves âgées. Les colonies sont inspectées journellement et des échantillons sont prélevés à intervalles réguliers pour déterminer l'état de développement des ovaires.

Le nombre d'ovarioles par ovaire est minimum chez mellifera $(3,2)$ et maximum chez capensis $(9,4)$. Un coefficient de régression caractéristique de chaque race a été trouvé en ce qui concerne la rapidité du développement ovarien (Fig. 6). Le degré de développement des ovaires est à peu près le mème pour toutes les races au moment du début de la ponte. La conséquence de ces deux faits est qu'il existe de grandes différences dans les temps de latence (Fig. 3) : 23-30 jours pour les races européennes, 16 jours pour adami de Crète (originaire du Moyen-Orient) et 5-10 jours pour les races africaines. Les différences dans les périodes de latence sont significatives, excepté pour certaines valeurs à l'intérieur du groupe africain.

Les ouvrières de mellifera pondent le plus petit nombre d'œufs, celles d'adami le plus grand. Il existe une corrélation négative entre le nombre d'œufs pondus et la rapidité de développement des ovaires, mais pas entre le nombre d'œufs pondus et le nombre d'ovarioles.

L'influence de l'âge des abeilles testées sur le potentiel de développement ovarien est faible et en général significatif seulement dans les classes d'âge élevées.

Ces résultats sont interprétés comme étant la conséquence de divers facteurs de répression génétique agissant sur le potentiel reproducteur des abeilles ouvrières. On discute de la valeur adaptative éventuelle de la reproduction arrhénotoque chez les ouvrières.

\section{SUMMARY}

SPECIFIC DIFFERENCES IN THE DEVELOPMENT OF OVARIES

AND EGG LAYING OF QUEENLESS WORKERS OF SEVERAL RACES OF THE HONEYBEE, APIS MELLIFERA L.

To determine worker bee reproductive potential the following parameters were studied in free flying colonies : number of ovarioles, course of ovary development, begin of oviposition (= latency period), eggs laid during the first 48 hours.

Bees of the following races were examined:

Apis mellifera carnica (C).

Apis mellifera ligustica (L).

Apis mellifera mellifera (M).

Apis mellifera adami (A).

Apis mellifera intermissa (IM).

Apis mellifera adansonit-scutellata (AD).

Apis mellifera capensis (CAP).

Incubator emerged worker bees were marked and introduced to small queenright carniolan colonies. Later the queen was caged and five days after caging the queen and all the bees from the recipient colony were removed. This left an experimental colony composed of about 1550 marked bees of a given race in age classes from 1 to 15 days and in the beginning, elder larvae. Colonies were controlled daily and sampled for ovary development at regular intervals.

Number of ovarioles per ovary was smallest in mellifera $(3,2)$ and greatest in capensis $(9,4)$. Regression coefficients characteristic of each race were found for rapidity of ovary development (fig. 6). Level of ovary development was about the same for all races at the beginning of oviposition. Great differences were found in the latency periods (fig. 3) : 23-30 days for European races, 
16 days for adami from Crete (originating from the Middle East) and 5-10 days for African races. Differences in latency periods are significant except for some of the values within the African group.

Worker bees of mellifera laid the smallest number of eggs, those of adami the most. Number of eggs laid was negatively correlated with the rapidity of ovary development, but not with ovariole numbers.

The influence of age of the experimental bees on the potential of ovary development was low being significant in most cases only in samples of the oldest bees.

These results are interpreted as the consequence of different genetic repressor factors acting on the reproductive potential of worker bees. The possible adaptive value of arrhenotokous reproduction by worker bees is discussed.

\section{LITERATURVERZEICHNIS}

ANDERson R. H., 1963. - The laying worker in the Cape honeybee, Apis mellifera capensis. J. Apic. Res., 2, 85-92.

BERLEPSCH A. von, 1873. - Die Biene und ihre Zucht mit beweglichen Waben. 2. Aufl., J. Schneider Verl. Mannheim.

Butler C.G., 1959. - The source of substance produced by a queen honeybee which inhibits development of the ovaries of the workers of her colony. Proc. Roy. Ent. Soc., A 34, 137-138.

Chaud-Netto J., Bueno O. C., 1979. - Number of ovarioles in workers of Apis mellifera adansonii and Apis mellifera ligustica: a comparative study. J. apic. Res., 18, 260-263.

Dejean A., Passera L., 1974. - Ponte des ouvrières et inhibition royale chez la fourmi Temnothorax recedens Nyl. (Formicidae, Myrmicinae, Hym.). Insectes Sociaux, 21, 343-356.

DreisCher H., 1955. - Untersuchungen über die Arbeitstätigkeit und Drüsenentwicklung altersbestimmter Bienen im weisellosen Volk. Zool. Jahrb. (Physiol.), 66, 429-472.

ENGELS W., 1974. - Occurence and significance of vitellogenins in female castes of social hymenoptera. Amer. Zool., 14, 1229-1237.

Engels W., FAhrenhorst H., 1974. - Alters- und kastenspezifische Veränderungen der HaemolymphProtein-Spektren bei A. mellifica. Wilhelm Roux'Archiv, 174, 285-296.

Fletcher D. J., 1978. - The African bee, Apis mellifera adansonii, in Africa. Ann. Rev. Entomol., 23, 151-171.

Gontarski (H.), 1938. - Beobachtungen an eierlegenden Arbeiterinnen. Disch. Imkerführer 12/4.

Hemmling C., Koeniger N., RuttNer F., 1979. - Quantitative Bestimmung der 9-Oxodecensäure im Lebenzyklus der Kapbiene (A. m. capensis Esch.) Apidologie, 10, 227-240.

Hess G., 1942. - Über den Einfluss der Weisellosigkeit und des Fruchtbarkeitsvitamins E auf die Ovarien der Bienenarbeiterin. Beih. Schweiz. Bienenzeitung Bd, 1, H. 2, 1-110.

Huber F., 1788. - Zit. nach v. Berlepsch 1873 und Gontarski 1938.

JAY S. C., 1968. - Factors influencing ovary development of worker honeybees under natural conditions. Can. J. Zool., 46, 345-347.

JAY S. C., 1975. - Factors influencing ovary development of worker honeybees of european and african origin. Can. J. Zool., 53, 1387-1390.

Lindauer M., 1952. - Ein Beitrag zur Arbeitsteilung im Bienenstaat. Z. vergl. Physiol., 34, 299-345.

MüssBichler A., 1952. - Die Bedeutung äusserer Einflüsse und der Corpora allata bei der Afterweiselentstehung von Apis mellifica. Z. vergl. Physiol., 34, 207-221.

Onions G. W., 1912. - South African "Fertile Worker Bees 》. Agric. J. Un. S. Afr., 3, 720-728.

PaIN J., 1955. - Influence des reines mortes sur le développement ovarien de jeunes ouvrières d'abeilles (Apis mellifica). Insectes Sociaux, 2, 35-43.

PerePelova B. J., 1926. - Biology of laying workers. Opytnisi Pasieka, 12, 8-10. 
RIEM, 1770. - Zït. nach Gontarski 1938.

RUTTNER F., 1977. - The problem of the Cape bee (Apis mellifera capensis Escholtz) : Parthenogenesis size of populations - evolution. Apidologie, 8, 281-294.

RuttNer F., Koeniger N., Veith H. J., 1976. - Queen substance bei eierlegenden Arbeiterinnen der Honigbiene (Apis mellifera L.). Naturwiss, 63, 434-435.

Sakagami S.F., Beig D., Zucchi R., AKahIRA Y., 1963. - Occurence of ovary-developed workers in queenright colonies of stingless bees. Rev. Brasil. Biol., 23, 115-129.

Velthuis H. H. V., 1970 a. - Ovarian development in Apis mellifera worker bees. Ent. Exp. and Appl., 13, 377-394.

Velthuis H. H.V., 1970 b. - Queen substances from the abdomen of the honeybee queen. Z. vergl. Physiol., 70, 210-222.

VooGD S., 1955. - Inhibition of ovary development in worker bees by extraction of the queen. Experientia, 19, $181-187$.

VoogD S., 1956. - The influence of the queen on the ovary development in workerbees. Experientia, 75, 199-201.

WeAver N., 1956. - Ovarian development of worker honey bees. J. econ. Entomol., 49, 854-857.

WeIss K., 1980. - Über die Entstehung der weiblichen Kasten im Bienenvolk. In Ruttner, Königinnenzucht, S. 25-44. Apimondia Verl. Bukarest.

WiLliams I. H., FreE J. B., 1975. - Effect of environmental conditions during the larval period on the tendency of worker honeybees to develop their ovaries (Apis mellifera: Hym, Apidae). J. Entomol. (A.), 49, 179-182.

Zander E., Löschel F., Meier K., 1916. - Die Ausbildung des Geschlechtes bei der Honigiene (Apis mellifica L.). Z. angew. Ent., 3, 1-74. 